\title{
Exportação de nutrientes e qualidade de cultivares de rosas em campo e em ambiente protegido
}

\author{
Roberto L Villas Bôas'; Leandro José G de Godoy²; Clarice Backes³; Claudinei Paulo de Lima³ Dirceu M \\ Fernandes ${ }^{1}$ \\ ${ }^{1}$ UNESP-FCA, Depto. Recursos Naturais, Ciência do Solo, C. Postal 237, 18610-907 Botucatu-SP (autor para correspondência); \\ ${ }^{2}$ UNESP-Unidade Diferenciada de Registro, R. Tamekishi Takano, 195, 11900-000 Registro-SP; ${ }^{3}$ Doutorandos UNESP-FCA; \\ rlvboas@fca.unesp.br
}

\section{RESUMO}

Foram avaliadas a exportação de nutrientes por cultivares de rosas e as características que refletem qualidade em campo e ambiente protegido. O delineamento experimental foi inteiramente casualizado, sendo os tratamentos constituídos por 15 e 12 cvs. em ambiente protegido e em campo, respectivamente, com três repetições. Cada unidade experimental foi constituída por duas plantas, sendo coletada uma haste por planta. Foram determinados o diâmetro da haste e do botão floral, comprimento da haste, fitomassa seca e acúmulo de macro e micronutrientes pelas hastes colhidas. Plantas que apresentaram hastes com maior comprimento e diâmetro apresentaram também maiores quantidades de nutrientes extraídos. Os nutrientes extraídos em maiores quantidades foram o $\mathrm{N} \mathrm{e}$ o K. Com base na quantidade de nutrientes extraídos é possível que se faça o agrupamento de cvs. para estabelecer uma recomendação de fertilizantes para as cvs. com exigência nutricional semelhante.

Palavras-chave: Rosa sp., nutrientes, extração, flores.

\begin{abstract}
Nutrients exportation and quality of rose cultivars in field and in a protected environment

The nutrient exportation from rose cultivars and characteristics that reflect quality in the field and in a protected environment were evaluated in this research. A completely randomized design was adopted, and in the protected environment the treatment was constituted of $15 \mathrm{cvs}$. and in the field of 12, with three repetitions. Each experimental unit consisted of two plants, being collected one branch per plant. Branches and flower bud diameter, branch length, dry matter and the macro and micronutrients contents in the branches were determined. Plants with greater length and diameter of branches also showed larger quantity of extracted nutrients. The nutrients taken in larger quantities were $\mathrm{N}$ and $\mathrm{K}$. Based on the amount of extracted nutrients, it is possible to establish groups of cvs. with similar nutritional requirement for the recommendation of fertilizers.
\end{abstract}

Keywords: Rosa $s p$., nutrients, extraction, flowers.

\section{(Recebido para publicação em 8 de agosto de 2007; aceito em 24 de setembro de 2008) (Received in January 3, 2007; accepted in July 9, 2008)}

$\mathrm{D}$ entre os produtos mais importantes da floricultura mundial, as rosas têm se destacado, movimentando valores da ordem de dez bilhões de dólares anualmente (Guterman, 2002). Embora existam flutuações no ranking mundial das flores de corte mais vendidas, a rosa sempre se posiciona entre as três mais procuradas (Daudt, 2002).

A floricultura brasileira vem conquistando o mercado externo, com aumento de $30 \%$ no valor da exportação de seus produtos em 2003, tendo o estado de São Paulo contribuído com $75 \%$ do valor da exportação total de US\$20 milhões (Santos \& Kiyuna, 2004). O Brasil é um grande produtor de rosas de corte, atendendo tanto o mercado externo como o interno, com grande potencial para aumento na produção (Novaro, 2005).

A rosa apresenta boa facilidade de cruzamentos, possibilitando gerar híbridos. O primeiro híbrido de rosa foi introduzido em 1867 e desde então mais de 10.000 variedades têm sido obtidas. Atualmente no Centre for Variety Research, Holanda, cerca de 2.800 híbridos têm sido submetidos ao registro de proteção de cultivares e esse número tem aumentado, em média, 80 variedades por ano (Esselink et al. 2003).

Os países que mais investem em pesquisas para obtenção de novas variedades são Holanda, Alemanha, Estados Unidos e Colômbia, patrocinadas geralmente por empresas privadas (Casarini et al., 2004). Segundo estes mesmos autores, atualmente, as variedades de rosas são distinguidas principalmente pela coloração das pétalas, forma do botão, tamanho das hastes, produtividade (hastes $\mathrm{m}^{-2}$ ) e resistência às doenças. O comprimento da haste é uma característica especial na comercialização de rosas de corte devido ao aumento do seu valor comercial de acordo com seu comprimento (Casarini, 2004).

Os consumidores e principalmente o mercado estrangeiro exigem quanti- dade e qualidade das rosas produzidas. Desse modo é necessário conhecer os aspectos mais importantes da produção, sendo que no Brasil existem poucos dados científicos sobre técnicas de produção de flores (Folegatti et al., 2001).

Dentre os tratos culturais envolvidos na produção de rosas, a nutrição das plantas assume um papel muito importante. O estudo de necessidade de nutrientes em rosas tem recebido atenção, pois é fundamental para conhecer as exigências em fertilizantes, que não provoquem aumento excessivo da condutividade elétrica do solo ou do substrato, promovendo equilíbrio adequado entre os nutrientes. Entretanto, para elaborar uma solução contendo nutrientes em quantidades ideais é necessário conhecer a absorção de cada elemento químico pela planta, relacionando-as com os fatores ambientais, substratos e características da espécie (Eymar et al., 1998).

Vale ressaltar que pode ocorrer uma diferença dentro da própria espécie, sen- 
Tabela 1. Comprimento da haste, diâmetro do caule e botão e matéria seca das diferentes cultivares de roseiras em cultivo protegido e em campo (branch length, stem and bud diameter and dry matter of different rose cultivars in field and protected environment). Botucatu, UNESP-FCA, 2007.

\begin{tabular}{|c|c|c|c|c|}
\hline \multirow[b]{2}{*}{ Cultivares } & \multicolumn{4}{|c|}{ Cultivo em ambiente protegido } \\
\hline & $\begin{array}{l}\text { Comprimento } \\
\text { da haste }(\mathrm{cm})\end{array}$ & $\begin{array}{c}\text { Diâmetro do } \\
\text { caule }(\mathrm{mm})\end{array}$ & $\begin{array}{l}\text { Diâmetro do } \\
\text { botão }(\mathrm{mm})\end{array}$ & $\begin{array}{c}\text { Matéria seca (g } \\
\left.\text { planta }^{-1}\right)\end{array}$ \\
\hline Adventure & $49,8 \mathrm{~b}$ & $7,5 \mathrm{a}$ & $28,5 \mathrm{~b}$ & $18,78 b$ \\
\hline Água & $45,7 \mathrm{~b}$ & $5,5 b$ & $28,9 \mathrm{~b}$ & $13,47 b$ \\
\hline Avalanche & $55,0 \mathrm{~b}$ & $5,9 \mathrm{~b}$ & 31,7 a & $18,61 \mathrm{~b}$ \\
\hline Bugnat & $50,3 \mathrm{~b}$ & $6,7 \mathrm{a}$ & 30,7 a & $17,89 \mathrm{~b}$ \\
\hline Carola & 63,4 a & $5,8 \mathrm{~b}$ & $28,7 \mathrm{~b}$ & $21,45 b$ \\
\hline El toro & $46,9 \mathrm{~b}$ & $6,3 \mathrm{~b}$ & $26,2 \mathrm{c}$ & $14,74 \mathrm{~b}$ \\
\hline Leonidas & 67,9 a & $8,1 \mathrm{a}$ & $26,1 \mathrm{c}$ & $32,33 \mathrm{a}$ \\
\hline Lipstique 1 & $50,9 \mathrm{~b}$ & $6,6 \mathrm{a}$ & $29,0 \mathrm{~b}$ & $16,38 \mathrm{~b}$ \\
\hline Lipstique 2 & $50,4 \mathrm{~b}$ & $5,9 \mathrm{~b}$ & 30,8 a & $15,57 \mathrm{~b}$ \\
\hline Marlicie & 65,2 a & $5,5 \mathrm{~b}$ & $23,8 d$ & $17,69 \mathrm{~b}$ \\
\hline Movistar & 69,3 a & $7,3 \mathrm{a}$ & $29,2 \mathrm{~b}$ & $27,31 \mathrm{a}$ \\
\hline Osiana & 68,3 a & 6,7 a & $27,1 \mathrm{c}$ & $17,73 \mathrm{~b}$ \\
\hline Vegas & 74,3 a & $6,8 \mathrm{a}$ & $25,9 \mathrm{c}$ & $26,63 a$ \\
\hline Virgínia & $61,9 a$ & $6,7 \mathrm{a}$ & $29,2 \mathrm{~b}$ & $24,74 \mathrm{a}$ \\
\hline Wow & $52,0 \mathrm{~b}$ & $5,5 \mathrm{~b}$ & $29,4 \mathrm{~b}$ & $12,75 \mathrm{~b}$ \\
\hline \multirow[t]{2}{*}{ CV (\%) } & 14,93 & 17,72 & 28,36 & 17,70 \\
\hline & \multicolumn{4}{|c|}{ Cultivo em campo } \\
\hline Cultivares & $\begin{array}{l}\text { Comprimento } \\
\text { da haste }(\mathrm{cm})\end{array}$ & $\begin{array}{c}\text { Diâmetro do } \\
\text { caule }(\mathrm{mm})\end{array}$ & $\begin{array}{l}\text { Diâmetro do } \\
\text { botão (mm) }\end{array}$ & $\begin{array}{c}\text { Matéria seca (g } \\
\text { planta-1) }\end{array}$ \\
\hline Ambiance & 68,6 a & $9,0 \mathrm{a}$ & $28,2 \mathrm{~d}$ & 37,89 a \\
\hline Carola & $62,6 \mathrm{~b}$ & $6,7 \mathrm{~b}$ & 37,6 a & $22,54 \mathrm{~b}$ \\
\hline Dallas & 74,8 a & $9,0 \mathrm{a}$ & $34,6 \mathrm{~b}$ & 33,95 a \\
\hline Dolores & 73,8 a & $7,5 \mathrm{~b}$ & $26,9 d$ & $22,97 \mathrm{~b}$ \\
\hline Lipstique & $61,9 \mathrm{~b}$ & $7,3 \mathrm{~b}$ & $33,6 \mathrm{~b}$ & $24,95 \mathrm{~b}$ \\
\hline Miracle & 68,3 a & $6,9 \mathrm{~b}$ & $27,5 d$ & $16,76 \mathrm{c}$ \\
\hline Osiana & 67,8 a & $7,5 \mathrm{~b}$ & $32,1 \mathrm{~b}$ & $27,76 \mathrm{~b}$ \\
\hline Racol & $50,9 \mathrm{c}$ & $6,2 \mathrm{~b}$ & $30,2 \mathrm{c}$ & $15,67 \mathrm{c}$ \\
\hline Texas & $65,8 \mathrm{~b}$ & $6,7 \mathrm{~b}$ & $32,6 \mathrm{~b}$ & $21,07 b$ \\
\hline Tiresk & $65,4 \mathrm{~b}$ & $6,7 \mathrm{~b}$ & $31,1 \mathrm{c}$ & $21,14 b$ \\
\hline Vegas & 70,8 a & $7,2 \mathrm{~b}$ & $31,6 \mathrm{~b}$ & $24,74 \mathrm{~b}$ \\
\hline Virgínia & $63,6 \mathrm{~b}$ & $6,2 \mathrm{~b}$ & $30,3 \mathrm{c}$ & $17,16 \mathrm{c}$ \\
\hline $\mathrm{CV}(\%)$ & 8,28 & 13,19 & 6,06 & 14,29 \\
\hline
\end{tabular}

Letras iguais na coluna não diferem entre si pelo teste de Scott-Knott a $5 \%$ de probabilidade (same letters in the column do not differ by Scott-Knott test, $5 \%$ of probability).

do que cada variedade apresenta uma necessidade nutricional diferenciada.

Na prática, a adubação feita em rosa tanto no campo como em cultivo protegido, segue um padrão, não havendo adubação específica para as cultivares, exceto quando ocorrerem deficiências observadas visualmente na planta. No entanto, observa-se que cultivares de rosa, além de gerarem hastes de tamanho diferenciado, também variam quanto ao número de hastes por $\mathrm{m}^{2}$.

O objetivo deste trabalho foi avaliar a exportação de nutrientes por cultiva- res de rosas e características que refletem qualidade em campo e ambiente protegido.

\section{MATERIAL E MÉTODOS}

O estudo consistiu da instalação de dois experimentos, em campo e em condições de ambiente protegido realizados em Holambra II, distrito de Paranapanema-SP.

No experimento em ambiente protegido utilizou-se uma estufa geminada do tipo Arco com cobertura de polietileno de baixa densidade (PEBD) transparente, com espessura de 0,15 $\mathrm{mm}$, aditivada contra raios ultravioleta e difusor de luz.

O delineamento experimental utilizado nos experimentos foi inteiramente casualizado, sendo os tratamentos constituídos por 15 cvs. de rosa em ambiente protegido e 12 em campo, com três repetições. Cada unidade experimental foi constituída por duas plantas, sendo coletada uma haste por planta.

As cultivares utilizados em ambiente protegido foram Adventure, Água, Avalanche, Bugnat, Carola, El toro, Leônidas, Lipstique 1, Lipstique 2, Marlicie, Movistar, Osiana, Vegas, Virgínia e Wow. Em campo: Ambience, Carola, Dallas, Dolores, Lipstique, Miracle, Osiana, Racol, Texas, Tiresk, Vegas e Virgínia.

O sistema de plantio, tanto em estufa como em campo, foi em canteiros de $0,15 \mathrm{~m}$ de altura, sendo as plantas espaçadas de 0,10 m e 1,0 m entre linhas.

Essas cultivares foram fertilizados através da fertirrigação, não havendo diferença na quantidade de fertilizante aplicado em função de cada variedade. A fertirrigação constituiu de duas fórmulas de fertilizantes específicas para duas fases de cultivo, uma de formação e outra de produção. Na fase vegetativa as quantidades de nutrientes aplicadas em $\mathrm{g}$ planta ${ }^{-1} \mathrm{ano}^{-1}$ foram: $10 \mathrm{~g}$ de $\mathrm{N}$, 2,70 g de $\mathrm{P}, 10 \mathrm{~g}$ de $\mathrm{K}_{2} \mathrm{O}, 2,70 \mathrm{~g}$ de $\mathrm{Ca}$, $0,90 \mathrm{~g}$ de $\mathrm{Mg}, 1,30 \mathrm{~g}$ de $\mathrm{S}, 0,05 \mathrm{~g}$ de $\mathrm{Zn}$, 0,013 g de $\mathrm{Cu}, 0,2 \mathrm{~g}$ de Fe, 0,06 g de $\mathrm{Mn}$ e $0,04 \mathrm{~g}$ de $\mathrm{B}$. Na fase reprodutiva aplicou-se 20; 5,50; 20 e 5,40 g planta $^{-1}$ $\mathrm{ano}^{-1}$ de $\mathrm{N}, \mathrm{P}, \mathrm{K}_{2} \mathrm{O}$ e Ca, respectivamente, sendo que para os demais nutrientes utilizou-se a mesma quantidade da fase vegetativa. $\mathrm{O}$ manejo da irrigação foi realizado fixando a freqüência de irrigação em dois dias.

No início do período de produção, os ramos basais foram podados para a brotação das hastes comerciais. No ponto de corte, momento em que as flores estão fechadas, não cerradas, mas apenas começando a abrir, realizou-se o corte de cada haste a partir do ponto de brotamento da mesma.

O comprimento da haste foi medido entre o ponto de corte até a base do botão utilizando uma régua graduada em 
centímetros; o diâmetro foi avaliado na parte média da haste e o botão na parte basal, ambos através de um paquímetro digital graduado em milímetros.

Após realização dessas medidas, as hastes foram lavadas para a eliminação de resíduos de agrotóxicos e poeira, acondicionadas em sacos de papel e secas em estufa de circulação de ar forçada por 72 horas na temperatura de $65^{\circ} \mathrm{C}$. Em seguida, o material foi pesado, determinada a fitomassa seca, moído e enviado para o laboratório da FCA para determinação da concentração de nutrientes, de acordo com a metodologia modificada de Malavolta et al. (1997). A quantidade de nutrientes extraídos por haste foi calculada pelo produto da fitomassa seca e da concentração dos mesmos.

Os resultados foram submetidos à análise de variância em cada experimento separadamente (ambiente protegido e campo) e as médias comparadas utilizando-se teste de Scott-Knott a 5\% utilizando o programa Sisvar versão 4.6 (Ferreira, 2003). As correlações foram feitas pelo método de Pearson.

\section{RESULTADOS E DISCUSSÃO}

Houve diferença entre o comprimento da haste das cultivares avaliadas, sendo que esses valores variaram de 45,7 a $74,8 \mathrm{~cm}$ quando cultivadas em ambiente protegido e de 50,9 a 70,8 cm quando cultivadas em campo (Tabela 1). Em ambiente protegido, as cultivares que apresentaram maior comprimento da haste foram Vegas, Movistar, Leonidas, Osiana, Marlice, Carola e Vírginia e em cultivo a campo: Dallas, Dolores, Vegas, Ambiance, Miracle e Osiana. Verificamse em ambos os experimentos, que as cultivares com maior comprimento da haste apresentam tamanho ideal ou próximo ao ideal exigido pelo mercado (70 cm) (Sebrae, 2006).

Casarini (2004) estudando a aplicação de N e K via fertirrigação em rosa, cultivar Versilha, observou que em todos os tratamentos os valores médios de comprimento de haste foram abaixo de $50 \mathrm{~cm}$, o que diminui o valor comercial da produção. Para a floricultura são necessários produtividade e qualidade do produto ofertado, sendo características

Tabela 2. Extração de macronutrientes pelos diferentes cultivares de roseiras em cultivo protegido e em campo (macronutrients extraction by different roses cultivars in a protected environment and in the field). Botucatu, UNESP-FCA, 2007.

\begin{tabular}{|c|c|c|c|c|c|c|}
\hline \multirow{3}{*}{ Cultivares } & \multicolumn{6}{|c|}{ Cultivo em ambiente protegido } \\
\hline & \multicolumn{6}{|c|}{ Extração de macronutrientes (mg haste ${ }^{-1}$ ) } \\
\hline & $\mathbf{N}$ & $\mathbf{P}$ & $\mathbf{K}$ & $\mathrm{Ca}$ & Mg & $\mathbf{s}$ \\
\hline Adventure & $231 \mathrm{c}$ & $24 \mathrm{~b}$ & $171 \mathrm{c}$ & $104 \mathrm{c}$ & $26 \mathrm{~b}$ & $29 a$ \\
\hline Água & $158 \mathrm{c}$ & $15 c$ & $118 \mathrm{c}$ & $58 \mathrm{c}$ & $15 \mathrm{c}$ & $18 \mathrm{~b}$ \\
\hline Avalanche & $217 c$ & $23 \mathrm{~b}$ & $170 \mathrm{c}$ & $87 \mathrm{c}$ & $18 \mathrm{~d}$ & 27 a \\
\hline Bugnat & $263 \mathrm{~b}$ & $24 b$ & $166 \mathrm{c}$ & $118 \mathrm{~b}$ & $28 \mathrm{~b}$ & $31 \mathrm{a}$ \\
\hline Carola & $251 \mathrm{~b}$ & 30 a & $164 \mathrm{c}$ & $98 \mathrm{c}$ & $21 \mathrm{c}$ & 29 a \\
\hline El toro & $181 \mathrm{c}$ & $22 \mathrm{~b}$ & $144 \mathrm{c}$ & $64 c$ & $16 \mathrm{c}$ & $20 \mathrm{~b}$ \\
\hline Leonidas & 392 a & $37 \mathrm{a}$ & $304 a$ & $188 \mathrm{a}$ & $44 a$ & $39 a$ \\
\hline Lipstique 1 & $199 \mathrm{c}$ & $21 \mathrm{~b}$ & $145 \mathrm{c}$ & $80 \mathrm{c}$ & $19 \mathrm{c}$ & $21 b$ \\
\hline Lipstique 2 & $212 c$ & $19 \mathrm{c}$ & $137 c$ & $121 \mathrm{~b}$ & $16 \mathrm{c}$ & $26 a$ \\
\hline Marlicie & $191 \mathrm{c}$ & $18 \mathrm{c}$ & $146 \mathrm{c}$ & $83 c$ & $26 \mathrm{~b}$ & $25 \mathrm{~b}$ \\
\hline Movistar & $299 \mathrm{~b}$ & $31 a$ & $215 b$ & $153 \mathrm{a}$ & $43 a$ & $34 \mathrm{a}$ \\
\hline Osiana & $185 \mathrm{c}$ & $23 \mathrm{~b}$ & $167 c$ & $58 c$ & $13 \mathrm{c}$ & $18 \mathrm{~b}$ \\
\hline Vegas & $289 \mathrm{~b}$ & $33 a$ & $244 b$ & $131 \mathrm{~b}$ & $30 \mathrm{~b}$ & $31 \mathrm{a}$ \\
\hline Virgínia & $282 \mathrm{~b}$ & $25 \mathrm{~b}$ & $237 \mathrm{~b}$ & $106 \mathrm{c}$ & $24 \mathrm{~b}$ & $26 a$ \\
\hline Wow & $158 \mathrm{c}$ & $18 \mathrm{c}$ & $126 \mathrm{c}$ & $54 \mathrm{c}$ & $16 \mathrm{c}$ & $19 \mathrm{~b}$ \\
\hline \multirow[t]{2}{*}{ CV\% } & 20,03 & 14,57 & 16,31 & 27,75 & 20,54 & 18,85 \\
\hline & \multicolumn{6}{|c|}{ Cultivo em campo } \\
\hline \multirow[t]{2}{*}{ Cultivares } & \multicolumn{6}{|c|}{ Extração de macronutrientes (mg haste ${ }^{-1}$ ) } \\
\hline & $\mathbf{N}$ & $\mathbf{P}$ & $\mathbf{K}$ & $\mathrm{Ca}$ & Mg & $\mathbf{S}$ \\
\hline Ambiance & 406 a & $46 \mathrm{a}$ & $345 \mathrm{a}$ & $181 \mathrm{a}$ & $32 \mathrm{~b}$ & $36 \mathrm{a}$ \\
\hline Carola & $215 b$ & $25 b$ & $174 \mathrm{~b}$ & $92 \mathrm{~b}$ & $15 d$ & $18 \mathrm{~b}$ \\
\hline Dallas & 343 a & 39 a & $228 b$ & $155 \mathrm{a}$ & 38 a & $32 \mathrm{a}$ \\
\hline Dolores & $234 \mathrm{~b}$ & $24 \mathrm{~b}$ & $206 \mathrm{~b}$ & $99 \mathrm{~b}$ & $31 \mathrm{~b}$ & $24 \mathrm{~b}$ \\
\hline Lipstique & $267 \mathrm{~b}$ & $25 \mathrm{~b}$ & $229 \mathrm{~b}$ & $144 \mathrm{a}$ & $30 \mathrm{~b}$ & $23 \mathrm{~b}$ \\
\hline Miracle & $191 \mathrm{~b}$ & $18 \mathrm{c}$ & $163 b$ & $86 \mathrm{~b}$ & $19 d$ & $15 \mathrm{~b}$ \\
\hline Osiana & $270 \mathrm{~b}$ & $39 a$ & $239 b$ & $108 \mathrm{~b}$ & $22 \mathrm{c}$ & $23 \mathrm{~b}$ \\
\hline Racol & $174 \mathrm{~b}$ & $15 c$ & $142 b$ & $87 \mathrm{~b}$ & $18 d$ & $18 b$ \\
\hline Texas & $208 \mathrm{~b}$ & $21 \mathrm{c}$ & $187 b$ & $71 \mathrm{~b}$ & $18 d$ & $18 b$ \\
\hline Tiresk & $267 \mathrm{~b}$ & $27 \mathrm{~b}$ & $195 \mathrm{~b}$ & $104 \mathrm{~b}$ & $24 \mathrm{c}$ & $21 b$ \\
\hline Vegas & $234 \mathrm{~b}$ & $30 \mathrm{~b}$ & $200 \mathrm{~b}$ & $96 \mathrm{~b}$ & $24 \mathrm{c}$ & $21 b$ \\
\hline Virgínia & $178 \mathrm{~b}$ & $20 \mathrm{c}$ & $155 \mathrm{~b}$ & $76 \mathrm{~b}$ & $12 \mathrm{~d}$ & $13 b$ \\
\hline $\mathrm{CV}(\%)$ & 17,87 & 17,81 & 17,97 & 21,19 & 16,92 & 21,74 \\
\hline
\end{tabular}

Letras iguais na coluna não diferem entre si pelo teste de Scott-Knott a 5\% de probabilidade (same letters in the column do not differ by Scott-Knott test, $5 \%$ of probability).

importantes no cultivo de rosas, o tamanho e cor do botão, a durabilidade, além do diâmetro e comprimento da haste (Aguirre, 2002).

Observou-se que houve diferença entre as cvs. quanto ao diâmetro da haste nas duas situações de cultivo (Tabela 1). Em ambiente protegido os maiores valores foram encontrados em Leonidas, Adventure, Movistar, Osiana, Vegas, Virgínia, Bugnat e Lipistique 1. No cultivo em campo as cultivares Ambience e Dallas apresentaram maiores diâmetros da haste $(9 \mathrm{~mm})$. Para Aguirre
(2002) a nutrição de roseira deve ser diferenciada para cada cultivar, pois cultivares com haste de maior diâmetro requerem maior quantidade de nutrientes. Este mesmo autor ressalta ainda a importância das hastes de maior diâmetro para aumento da durabilidade da flor após a colheita.

Houve correlação entre o diâmetro da haste e a quantidade de macronutrientes extraídos pela planta, demonstrando que cultivares com maior diâmetro necessitam de maiores quantidades de nutrientes conforme observa- 
Tabela 3. Extração de micronutrientes pelos diferentes cultivares de roseiras em cultivo protegido e em campo (micronutrients extraction by different roses cultivars in a protected environment and in the field). Botucatu, UNESP-FCA, 2007.

\begin{tabular}{|c|c|c|c|c|c|}
\hline \multirow{3}{*}{ Cultivares } & \multicolumn{5}{|c|}{ Cultivo em ambiente protegido } \\
\hline & \multicolumn{5}{|c|}{ Extração de micronutrientes $\left(\mu \mathrm{g}\right.$ haste $\left.^{-1}\right)$} \\
\hline & B & $\mathrm{Cu}$ & $\mathrm{Fe}$ & Mn & $\mathrm{Zn}$ \\
\hline Adventure & $0,25 \mathrm{~b}$ & $0,12 \mathrm{c}$ & $2,17 \mathrm{~b}$ & 2,72 & $1,56 \mathrm{~b}$ \\
\hline Água & $0,23 \mathrm{~b}$ & $0,07 \mathrm{c}$ & $1,64 \mathrm{c}$ & 1,94 & $0,95 \mathrm{~b}$ \\
\hline Avalanche & $0,37 \mathrm{~b}$ & $0,09 \mathrm{c}$ & $1,78 b$ & 2,68 & $1,40 \mathrm{~b}$ \\
\hline Bugnat & $0,43 \mathrm{~b}$ & $0,14 \mathrm{c}$ & $1,81 b$ & 2,42 & $1,55 \mathrm{~b}$ \\
\hline Carola & $0,44 \mathrm{~b}$ & $0,13 \mathrm{c}$ & $1,55 \mathrm{c}$ & 2,83 & $1,65 \mathrm{~b}$ \\
\hline El toro & $0,25 \mathrm{~b}$ & $0,07 \mathrm{c}$ & $1,18 \mathrm{c}$ & 2,04 & $1,30 \mathrm{~b}$ \\
\hline Leonidas & $0,72 \mathrm{a}$ & $0,29 \mathrm{~b}$ & $2,88 \mathrm{a}$ & 4,21 & $3,03 \mathrm{a}$ \\
\hline Lipstique 1 & $0,30 \mathrm{~b}$ & $0,08 \mathrm{c}$ & $1,55 \mathrm{c}$ & 1,96 & $1,18 b$ \\
\hline Lipstique 2 & $0,34 \mathrm{~b}$ & $0,08 \mathrm{c}$ & $2,02 \mathrm{~b}$ & 2,97 & $1,43 \mathrm{~b}$ \\
\hline Marlicie & $0,26 \mathrm{~b}$ & $0,70 a$ & $1,38 \mathrm{c}$ & 2,74 & $1,54 \mathrm{~b}$ \\
\hline Movistar & $0,69 a$ & $0,24 \mathrm{~b}$ & $2,07 \mathrm{~b}$ & 2,91 & $1,89 \mathrm{~b}$ \\
\hline Osiana & $0,36 \mathrm{~b}$ & $0,29 b$ & $0,75 \mathrm{c}$ & 0,98 & $1,09 \mathrm{~b}$ \\
\hline Vegas & $0,64 a$ & $0,13 c$ & $2,16 \mathrm{~b}$ & 3,00 & $1,75 b$ \\
\hline Virgínia & $0,45 \mathrm{~b}$ & $0,34 \mathrm{~b}$ & $1,34 \mathrm{c}$ & 1,84 & $1,48 b$ \\
\hline Wow & $0,25 \mathrm{~b}$ & $0,09 \mathrm{c}$ & $1,15 \mathrm{c}$ & 2,31 & $1,26 \mathrm{~b}$ \\
\hline \multirow[t]{2}{*}{ CV\% } & 29,56 & 29,00 & 24,44 & 26,57 & 23,88 \\
\hline & \multicolumn{5}{|c|}{ Cultivo em campo } \\
\hline \multirow[t]{2}{*}{ Cultivares } & \multicolumn{5}{|c|}{ Extração de micronutrientes $\left(\mu \mathrm{g}_{\text {haste }}{ }^{-1}\right)$} \\
\hline & B & $\mathrm{Cu}$ & Fe & Mn & $\mathrm{Zn}$ \\
\hline Ambiance & $0,64 \mathrm{a}$ & $1,44 \mathrm{a}$ & $1,69 \mathrm{a}$ & $1,37 \mathrm{a}$ & $2,46 a$ \\
\hline Carola & $0,37 \mathrm{c}$ & $0,64 \mathrm{c}$ & $0,79 \mathrm{c}$ & $1,51 \mathrm{a}$ & $1,31 \mathrm{~b}$ \\
\hline Dallas & $0,50 \mathrm{~b}$ & $1,04 b$ & $1,53 \mathrm{a}$ & $1,61 \mathrm{a}$ & $1,54 \mathrm{~b}$ \\
\hline Dolores & $0,35 \mathrm{c}$ & $0,57 \mathrm{c}$ & $1,13 b$ & $0,61 \mathrm{~b}$ & $1,01 \mathrm{~b}$ \\
\hline Lipstique & $0,40 \mathrm{c}$ & $0,83 \mathrm{c}$ & $1,24 \mathrm{~b}$ & $0,61 \mathrm{~b}$ & $1,43 b$ \\
\hline Miracle & $0,23 d$ & $0,67 c$ & $0,74 \mathrm{c}$ & $0,76 \mathrm{~b}$ & $1,18 \mathrm{~b}$ \\
\hline Osiana & $0,64 \mathrm{a}$ & $0,70 \mathrm{c}$ & $1,14 \mathrm{~b}$ & $1,67 \mathrm{a}$ & $1,22 b$ \\
\hline Racol & $0,29 \mathrm{~d}$ & $0,41 \mathrm{c}$ & $0,77 \mathrm{c}$ & $0,42 \mathrm{~b}$ & $0,72 b$ \\
\hline Texas & $0,41 \mathrm{c}$ & $0,67 \mathrm{c}$ & $1,24 \mathrm{~b}$ & $1,61 \mathrm{a}$ & $0,99 \mathrm{~b}$ \\
\hline Tiresk & $0,33 d$ & $0,49 \mathrm{c}$ & $1,07 \mathrm{~b}$ & $0,58 \mathrm{~b}$ & $1,09 \mathrm{~b}$ \\
\hline Vegas & $0,44 \mathrm{c}$ & $0,41 \mathrm{c}$ & $1,19 \mathrm{~b}$ & $0,59 \mathrm{~b}$ & $0,84 \mathrm{~b}$ \\
\hline Vergília & $0,27 d$ & $0,69 \mathrm{c}$ & $0,77 \mathrm{c}$ & $0,56 \mathrm{~b}$ & $0,90 \mathrm{~b}$ \\
\hline $\mathrm{CV}(\%)$ & 19,14 & 28,05 & 20,26 & 25,89 & 24,07 \\
\hline
\end{tabular}

Letras iguais na coluna não diferem entre si pelo teste de Scott-Knott a $5 \%$ de probabilidade. (same letters in the column do not differ by Scott-Knott test, $5 \%$ of probability).

ções de Aguirre (2002). Todas as correlações foram significativas a $1 \%$, sendo que os valores de $\mathrm{R}^{2}$ para o cultivo protegido foram de 0,$77 ; 0,74 ; 0,77 ; 0,72$; 0,72 e 0,66 para N, P, K, Ca, Mg e S, respectivamente. Para o cultivo em campo foram observados os valores de $\mathrm{R}^{2}$ : $\mathrm{N}=0,92 ; \mathrm{P}=0,87 ; \mathrm{K}=0,84 ; \mathrm{Ca}=0,88$; $\mathrm{Mg}=0,87$ e $\mathrm{S}=0,95$.

Para o cultivo em campo a cultivar Carola apresentou maior diâmetro de botão $(37,6 \mathrm{~mm})$, quando comparada às demais, sendo que para essa mesma cultivar em ambiente protegido o diâmetro do bo-
Verifica-se que houve diferença entre as cultivares em relação às quantidades de macro e micronutrientes nas haste nos dois experimentos (Tabelas 2 e 3). Em ambiente protegido a cultivar Leonidas apresentou maior acúmulo de nitrogênio. Para os demais macronutrientes, outras cultivares igualaram-se estatisticamente.

Cabrera (2002) avaliando a cultivar Bridal Wite sobre quatro porta-enxertos ( $R$. manetti, $R$. x odorata, $R$. x Natal Briar e $R$. x Dr. Huey) verificou diferença entre as mesmas nos teores de $\mathrm{P}, \mathrm{K}, \mathrm{Mg}, \mathrm{Mn}, \mathrm{Fe}$, B e Zn podendo dessa forma influenciar na quantidade de nutrientes extraídos pelas plantas, ressaltando a necessidade nutricional diferenciada, mesmo dentro da mesma espécie.

No cultivo em campo, em geral, as cultivares Ambience e Dallas extraíram maiores quantidades dos nutrientes, correspondendo também às cultivares que produziram maior fitomassa seca.

A extração média dos macronutrientes (mg haste ${ }^{-1}$ ) pelas cultivares em ambiente protegido e em campo foi: $\mathrm{N}(234)>\mathrm{K}(177)>\mathrm{Ca}(100)$ $>\mathrm{P}(26)>\mathrm{Mg}(24)=\mathrm{S}(24)$ e N (249) > $\mathrm{K}(205)>\mathrm{Ca}(108)>\mathrm{P}(27)>\mathrm{Mg}(24)$ $>\mathrm{S}$ (22), respectivamente. Para os micronutrientes a média em $\mu \mathrm{g}$ haste ${ }^{-1}$ no cultivo protegido foi: $\mathrm{Mn}(2,50)>\mathrm{Fe}$ $(1,70)>\mathrm{Zn}(1,54)>\mathrm{B}(0,40)>\mathrm{Cu}$ $(0,19)$. Em campo: $\mathrm{Zn}(1,22)>\mathrm{Fe}(1,11)$ $>\mathrm{Mn}(0,99)>\mathrm{Cu}(0,71)>\mathrm{B}(0,41)$.

Os nutrientes mais exportados pelas plantas foram o $\mathrm{N}$ e $\mathrm{K}$, que segundo Casarini et al. (2004) são os nutrientes exigidos em maiores quantidades pelas rosas, pois apresentam funções importantes e distintas no desenvolvimento da roseira. $\mathrm{O}$ nitrogênio é absorvido em maior quantidade na fase de crescimento vegetativo quando a planta desenvolve mais massa foliar e reservas de carboidratos que posteriormente serão usados na formação de ramos basais, formando assim o esqueleto da roseira; já o potássio é absorvido em maior quantidade na fase de desenvolvimento do botão floral conferindo tamanho e coloração às pétalas (qualidade da floração).

A variação na quantidade de nutrientes absorvidos sugere que as cultivares 
devam receber adubações específicas. Se for dividido o maior valor de extração pelo menor tem-se para cada nutriente a variação máxima obtida entre cultivares foi de: N 2,6, P 3,0, K 2,9, Ca 3,5, $\mathrm{Mg} \mathrm{3,6}$ e S 2,9 vezes. Apesar dos valores de extração serem maiores para $\mathrm{N}$ e $\mathrm{K}$, para $\mathrm{Mg}$, nutriente em relação ao qual é muito comum de se observar deficiência em roseiras, as diferenças são maiores e uma complementação foliar com esse nutriente parece ser uma alternativa para as culturas.

Segundo Padilha (1999) a extração de nutrientes depende do tamanho da planta (grande, médio e pequeno), onde as maiores extraem as seguintes quantidades de N, P e K: 19,2; 5,5 e 20,9 g planta $^{-1}$ ano $^{-1}$, respectivamente. Com uma produção de 54 hastes planta ${ }^{-1}$ ano $^{-1}$, essas quantidades correspondem a 354 mg haste ${ }^{-1}$ de $\mathrm{N}, 102 \mathrm{mg}$ haste $^{-1}$ de $\mathrm{K} \mathrm{e}$ $386 \mathrm{mg}$ haste $^{-1}$ de K. Verifica-se na Tabela 2 que a quantidade de $\mathrm{N}$ extraída pela cultivar Dallas em campo (343 mg haste $^{-1}$ ) é semelhante à encontrado por Padilha (1999); entretanto as cultivares Leonidas em ambiente protegido e Ambience em campo apresentaram valores maiores de $\mathrm{N}$ exportados, 392 e $406 \mathrm{mg}$ haste $^{-1}$, respectivamente. Verifica-se também que as quantidades de $\mathrm{P}$ e $\mathrm{K}$ extraídas pelas hastes foram inferiores aos dados observados por Padilha (1999).

Em relação aos demais nutrientes, os níveis requeridos pela planta por ano são 5,41; 1,71 e 1,30 0,04; 0,013; 0,194 e 0,06 g planta ${ }^{-1}$ para $\mathrm{Ca}, \mathrm{Mg}, \mathrm{S}, \mathrm{B}, \mathrm{Cu}$, $\mathrm{Fe}$ e Mn, respectivamente.

Considerando apenas a haste colhida e uma produção média de 100 hastes por $\mathrm{m}^{2}$ por ano, tem-se que a necessidade mínima de fertilizante a ser aplicada seria de $406 \mathrm{~kg} \mathrm{ha}^{-1}$ de N, $105 \mathrm{~kg} \mathrm{ha}^{-1}$ de $\mathrm{P}_{2} \mathrm{O}_{5}, 413 \mathrm{~kg} \mathrm{ha}^{-1}$ de $\mathrm{K}_{2} \mathrm{O}, 180 \mathrm{~kg} \mathrm{ha}^{-1}$ de $\mathrm{Ca}, 44 \mathrm{~kg} \mathrm{ha}^{-1}$ de $\mathrm{Mg}$ e $38 \mathrm{~kg} \mathrm{ha}^{-1}$ de $\mathrm{S}$. Tamimi \& Matsuyama (1999) utilizan- do a cultivar Odorata no Havaii verificaram uma remoção de $256,2 \mathrm{~kg} \mathrm{ha}^{-1} \mathrm{de}$ $\mathrm{N}, 30,0 \mathrm{~kg} \mathrm{ha}^{-1}$ de P, $187,5 \mathrm{~kg} \mathrm{ha}^{-1}$ de $\mathrm{K}$, $116,3 \mathrm{~kg} \mathrm{ha}^{-1}$ de $\mathrm{Ca}, 26,0 \mathrm{~kg} \mathrm{ha}^{-1}$ de $\mathrm{Mg}$ e $21,1 \mathrm{~kg} \mathrm{ha}^{-1}$ de $\mathrm{S}$ com uma densidade de 10 plantas por $\mathrm{m}^{2}$ gerando uma população de 61.775 plantas por hectare e produção média de 30 hastes por planta por ano.

Como não é possível estabelecer uma recomendação de fertilizantes para cada cultivar, uma solução seria o agrupamento das cultivares em exigências diferenciadas.

Com base na extração de nutrientes pelas diferentes cultivares, as mesmas poderiam ser agrupadas em quatro categorias de acordo com exigência nutricional: muito alta, alta, média e baixa. Em ambiente protegido: muito alta: Leonidas, Movistar e Vegas; alta: Carola e Virgínia; média: Adventure, Avalanche, Bugnat, Lipstique 1, Lipstique 2, Marlicie e Osiana; baixa: Água, El toro e Wow. Em campo: muito alto: Ambience e Dallas; alto: Lipstique, Osiana e Vegas; médio: Carola, Dolores, Texas Tirex; baixo: Miracle, Racol e Virgínia.

\section{REFERÊNCIAS}

AGUIRRE CEC. 2007. 02 de abril. Nutrición Vegetal in flor de corte en el sur del estado de México. Disponível em http://www.uaaan.mx/ academic/Horticultura/Memhort02/ Ponencia08.pdf.

CABRERA RI. 2002. Rose yield, dry matter partitioning and nutrient status responses to rootstock selection. Scientia Horticulturae 95: 75-83.

CASARINI E. 2004. Doses de $N$ e $K$ aplicados via fertirrigação na cultura da roseira (Rosa sp.) em ambiente protegido. Piracicaba: USPESALQ, 101p. (Tese doutorado).

CASARINI E; FOLEGATTI MV; ARTIGIANE VH. 2004. Fertirrigação em rosas In: BOARETTO AE; VILLAS BÔAS RL; SOUZA WF; PARRA IRV. Fertirrigação teorias e práticas. CDROM, Piracicaba, p. 370-389.
DAUDT RHS. 2002. Censo da Produção de Flores e Plantas Ornamentais no RS na Virada do Milênio. Rio Grande do Sul: UFRGSFaculdade de Agronomia, 107p. (Tese mestrado).

EYMAR ALONSO E; LÓPEZ VELA D; CADAHÍA LÓPEZ C.1998. Fertirrigacion de coniferas y rosal. In: CADAHÍA LOPEZ C. Fertirrigacion: cultivos hortícolas $y$ ornamentales. Madrid: Mundi-Prensa, 1998. Ap. 2, p. 417-463.

ESSELINK G; SMULDERS M; VOSMAN B. 2003. Identification of cut rose (Rosa hybrida) and rootstock varieties using robust sequence tagged microsatellite site markers. Theor Appl Genet 106: 277-286.

FERREIRA DF. 2003. Sisvar versão 4.2. DEX/ UFLA.

FOLEGATTI MV; CASARINI E; BLANCO FF. 2001. Greenhouse irrigation water depths in relation to rose stem and bud qualities. Scientia Agricola 58: 465-468.

GUTERMAN I. 2002. Rose Scent - genomic approaches to discovering novel floral fragrance - related genes. Plant Cell 14: 23252338.

MALAVOLTA E; VITTI GC; OLIVEIRA SA. 1997. Avaliação do estado nutricional das plantas: princípios e aplicações, $2 \mathrm{ed}$. Piracicaba: POTAFOS (Associação brasileira de pesquisa da potassa e do fosfato), 317p.

NOVARO N. 2005. Breeders rights and Brazilian roses. FloraCulture International 15: 32.

PADILLA WA. 1999. El uso de la fertirrigacion en cultivos de flores en latinoamerica. In: FOLEGATTI MV Coord. Fertirrigação: Citrus, Flores, Hortaliças. Guaíba: Agropecuária, Cap. 5, p.355-384.

SANTOS VF; KIYUNA I. 2004. Floricultura do Estado de São Paulo: Novas fronteiras. In: XLII CONGRESSO BRASILEIRO DE ECONOMIA E SOCIOLOGIA RURAL, Anais eletrônicos... Disponível em http:// w w w. i e a.s p.g o v. b r / o u t / verTexto.php?codTexto=1470. Acessado em 20 de maio de 2007.

SEBRAE. Ponto de Partida: Cultivo de Rosas. Disponível em: <http:// www.biblioteca.sebrae.com.br/bte/bte.nsf/ DowContadar?OpenAgent\&unid=9B8DC19ABD15216B03256F F10049CC21>. Acesso em 21 mar. 2006.

TAMIMI YN; MATSUYAMA DT. 1999. Distribution of nutrients in CUT-flower roses and the quantities of biomass and nutrients removed during harvest. HortScience 34: 251253.

URCULLU GB. 1953 Cultivos ornamentales para flor cortada: Cultivo del rosal. Barcelona: Salvat, 512p. 